\title{
Study on Growth Habit, SHG and Thermal Properties of Urea Phosphate Doped KDP Crystals
}

\author{
G. G. Muley
}

Department of Phy sics, Sant Gadge Baba Amravati University, Amravati, 444602, (MS) India

\begin{abstract}
Pure and doped with Urea Phosphate single crystals of Potassium Dihydrogen Phosphate (KDP) were grown by the low temperature solution growth method, adopting the technique of slow evaporation of the solvent for nonlinear optical applications. The change in the growth habit of the doped KDP crystals has been reported. The study confirms the decrease in the growth rate along c-axis with increasing the dopant concentration. The doping of the Urea Phosphate in the grown crystal has been confirmed qualitatively by the FT-IR spectroscopy. Form the UV-Visible-NIR transmission study; it was observed that the transparency of the crystal increases with dopant concentration. Thermo Gravimetric Analysis shows that the thermal stability of the crystal decreases with dopant concentration. The crystal structure of doped crystal was determined from Powder X-Ray diffraction studies. The improvement in Second Harmonic Generation efficiency of doped crystal has also been reported.
\end{abstract}

Keywords NLO crystal, Growth habit, Second harmonic generation, XRD study

\section{Introduction}

Potassium Dihydrogen Phosphate (KDP) crystal is most widely used and thoroughly studied nonlinear optical (NLO) crystal. The attempts have been made to modify the properties favorable for NLO applications and growth rate of the KDP crystal by either changing the growth conditions or by adding different impurities[1-6]. The Ethylenediamine tetra acetic acid (EDTA) inhibits growth of KDP and acts as scattering centers in grown crystals[7] while on the other side it prevents number of nucleation's, increasing metastable zone width, which helps in growing big size good quality crystal[8]. Azo-organic dye A maranth gets absorbed and colors the pyramidal section $\left(\begin{array}{lll}1 & 0 & 1\end{array}\right)$ of the crystals and Sunset yellow FCF modifies the crystal habit and color of KDP. It decreases size of prismatic section of the crystal[9]. The increase in mean growth rate along the[001] direction has been reported with an increase in the concentration of trivalent impurity $\mathrm{Cr}(\mathrm{III})$ in the $\mathrm{KDP}$ solution while the growth rate along[100] direction is not altered[10]. A similar effect of $\mathrm{Fe}(\mathrm{III})$ and $\mathrm{Cr}(\mathrm{III})$ on the growth rate of KDP crystals has been reported by Owczarek and Sangwal[11]. Amino acids have very large optical nonlinearity, assigned to the chiral structure, used by many workers to imp rove the NLO, optical, thermal and mechanical properties of KDP using as a dopant[12,13].

* Corresponding author:

gajananggm@yahoo.co.in (G. G. Muley)

Published online at http://journal.sapub.org/scit

Copyright @ 2012 Scientific \& Academic Publishing. All Rights Reserved
Urea has been reported as a NLO material. Although it's optical and mechanical properties are comparable to those of KDP, but its crystallization and handling is rather difficult as it is highly hygroscopic. Different derivatives of urea have been studied for NLO applications. Some of them found useful for NLO applications[6,14-18]. The Urea and Urea derivates Thiourea, N'N dimethyl Urea have been tried as a dopent in KDP[19-22].

As KDP is being widely used in NLO applications and demands to modify its properties, the research work on the modifications of the optical, thermal, mechanical and NLO properties and the growth rate of the KDP crystal by using different dopants is still going on[23-25]. The urea and urea derivatives have high NLO efficiency and can be used as dopant to modify the properties of KDP crystal. In the present work, an attempt has been made to catch the properties of Urea derivative; Urea Phosphate and add it with KDP to investigate the effects. The growth of Urea Phosphate doped KDP crystals and their characterizations have been presented. Improvement in the SHG efficiency has been reported. Modification in the growth habit has also been observed.

\section{Experimental}

\subsection{Bulk Crystal Growth}

The solubility's of the pure (KDP) and $2 \mathrm{~mol} \%$, $4 \mathrm{~mol} \%$ and 6mol\% Urea Phosphate doped KDP (KU1, KU2 and KU3 respectively) crystals were measured gravimetrically in double distilled water in the temperature range $30-60^{\circ} \mathrm{C}$ 
(Figure 1). The materials have positive solubility gradient with temperature in water. The double distilled water was used as a solvent throughout the experiment.

Good quality crystals of pure and doped KDP were grown within 3-4 weeks. In doped crystals, growth rate along c-axis is found to be decreased. The $\mathrm{pH}$ of the solutions was ranged from 4.5 to 4.7. The photographs of some of the grown crystals are shown in Figure 2.

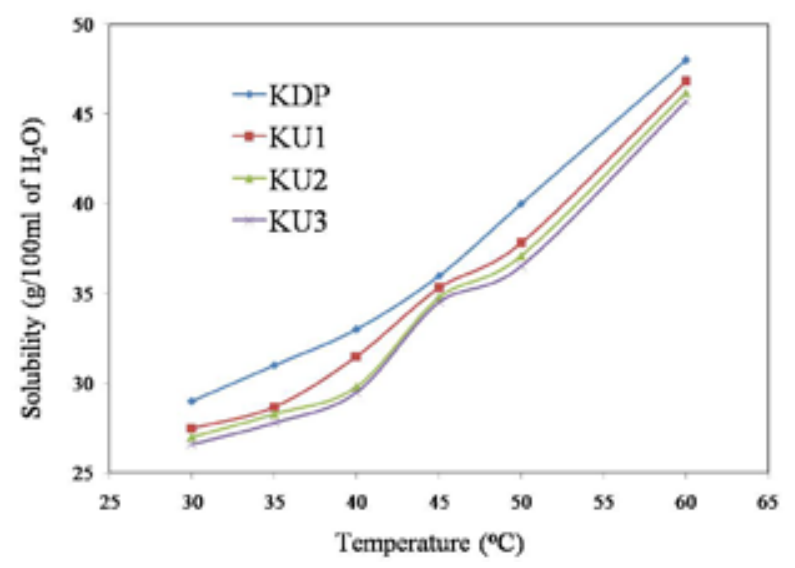

Figure 1. Solubility curves

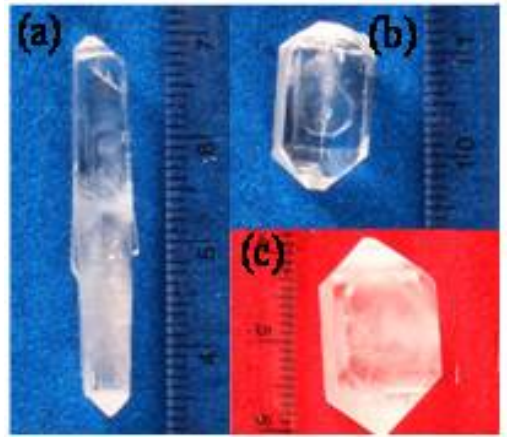

Figure 2. Photographs of (a) KDP, (b) KU1, (c) KU3 crystals

\subsection{Char acterization}

The FT-IR study was carried on the pure and doped KDP crystals to confirm qualitatively, the doping of the dopants in the crystals. Transparency of the crystals in the wavelength range $190-1100 \mathrm{~nm}$ was studied by using UV-1700 SHIMADZU Spectrophotometer. Powder XRD patterns were recorded on XPERT Pro Diffractometer and analyzed by Powder X software. The results have been presented. SHG efficiency was measured by employing Kurtz and Perry method. The SEM images of the crystals on the pyramidal section (1 01 1) were taken.

\section{Results and Discussion}

\subsection{Growth Habit}

The morphology of KDP crystals has tetragonal pris matic (100) and (010) faces and two tetragonal pyramids with the faces (101) and (011). The chemical bonds formed in KDP crystal between the growth units are the ones between $\mathrm{K}^{+}$ cations and $\mathrm{H}_{2} \mathrm{PO}_{4}^{-}$anions, as well as the hydrogen bonds of the adjacent $\mathrm{H}_{2} \mathrm{PO}_{4}^{-}$groups. The prismatic faces of KDP grow by stacking positive $\mathrm{K}^{+}$and negative $\mathrm{H}_{2} \mathrm{PO}_{4}{ }^{-}$ions alternately leaving the face $\{100\}$ charged neutrally and adsorbs the metal cations and organic molecules, which can form hydrogen bonds. The pyramidal growth sectors $\{101\}$ are stacked in the mode of two layers of cations and two layers of anions alternately. Each layer consists completely of either cations or anions. The potassium ions join into the lattice site rapidly as the negatively charged double layer $\left(\mathrm{H}_{2} \mathrm{PO}_{4}{ }^{-}\right)$is formed, because of its small volume, light weight and no preferred orientation required as compared with $\mathrm{H}_{2} \mathrm{PO}_{4}^{-}$. Thus, the pyramidal face is always positively charged throughout the growth process. The anions get adsorbed on the positively charged (101) planes and continues growth of the crystal in the direction[26]. It has evidences that the metallic cations and dyes influence the growth of prismatic (100) section and pyramidal (101) section of KDP crystals[27-31].

In the present study, the growth rate of doped crystals along c-axis is found to be decreased. In the Urea Phosphate doped KDP crystals, the decrease in the growth rate along c-axis, is possibly because of accommodations of the bigger size an ion $\mathrm{CO}\left(\mathrm{NH}_{2}\right)_{2} \mathrm{H}_{2} \mathrm{PO}_{4}{ }^{-}$on the pyramidal section at the place of relatively smaller size anion $\mathrm{H}_{2} \mathrm{PO}_{3}{ }^{2-}$, moreover two groups; $\mathrm{CO}\left(\mathrm{NH}_{2}\right)_{2}$ and $\mathrm{H}_{2} \mathrm{PO}_{4}{ }^{-}$are bounded by hydrogen bonding, have less affinity to other anions $\mathrm{CO}\left(\mathrm{NH}_{2}\right)_{2} \mathrm{H}_{2} \mathrm{PO}_{4}{ }^{-}$as compared to the $\mathrm{H}_{2} \mathrm{PO}_{4}{ }^{-}$groups in pure $\mathrm{KDP}$, as a result, growth rate decreases along[001] direction. In the SEM image (Figure 3) of the pure KDP, the surface appears rough as compared to the doped crystal, showing more sites for the attachments of the ions; cations and anions.

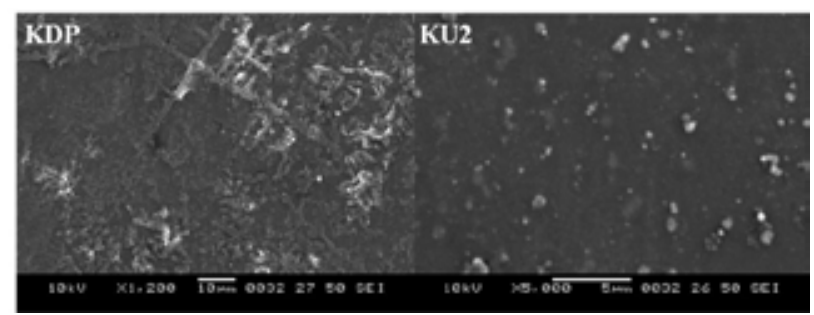

Figure 3. SEM images of the (101) plane

\subsection{Fourier Transform Infrared (FT-IR) Study}

The FT-IR spectra of pure and doped KDP crystals (Figure 4) were recorded on Perkin Elmer FT-IR Spectrophotometer within the wavenumber range 600 to $4000 \mathrm{~cm}^{-1}$.

In the FTIR spectrum of pure KDP crystal, the observed absorption peak at $3734.1 \mathrm{~cm}^{-1}$ corresponds to the $\mathrm{P}-\mathrm{OH}$ stretching, $2365.12 \mathrm{~cm}^{-1}$ to $\mathrm{O}-\mathrm{H}$ and $\mathrm{P}-\mathrm{OH}$ stretching, $1750.24 \mathrm{~cm}^{-1}$ to the P-O-H bending, $1279.19 \mathrm{~cm}^{-1}$ to O-H deformation and $\mathrm{P}=\mathrm{O}$ stretching and $915.2 \mathrm{~cm}^{-1}$ attributed to $\mathrm{P}-\mathrm{OH}$ stretching and $\mathrm{HO}-\mathrm{P}-\mathrm{OH}$ bending.

In the FT-IR spectra of Urea Phosphate doped KDP crystals, same peaks with slight change in positions due to hydrogen bonding have been observed with some additional peaks. These additional peaks observed in the range 3000 $\mathrm{cm}^{-1}-2800 \mathrm{~cm}^{-1}$ corresponds to the C-H stretching. The peaks 
at around $1300 \mathrm{~cm}^{-1}$ are attributed to the $\mathrm{O}-\mathrm{H}$ deformation and $\mathrm{P}=\mathrm{O}$ stretching, $\mathrm{C}-\mathrm{H}$ Deformation, $\mathrm{C}=\mathrm{O}$ and $\mathrm{N}-\mathrm{H}$ Stretching. The peaks $1100 \mathrm{~cm}^{-1}$ are as signed to the $\mathrm{C}-\mathrm{H}, \mathrm{O}-\mathrm{H}$ Deformation and C-O Stretching, which confirms the doping of the crystals with Urea Phosphate.

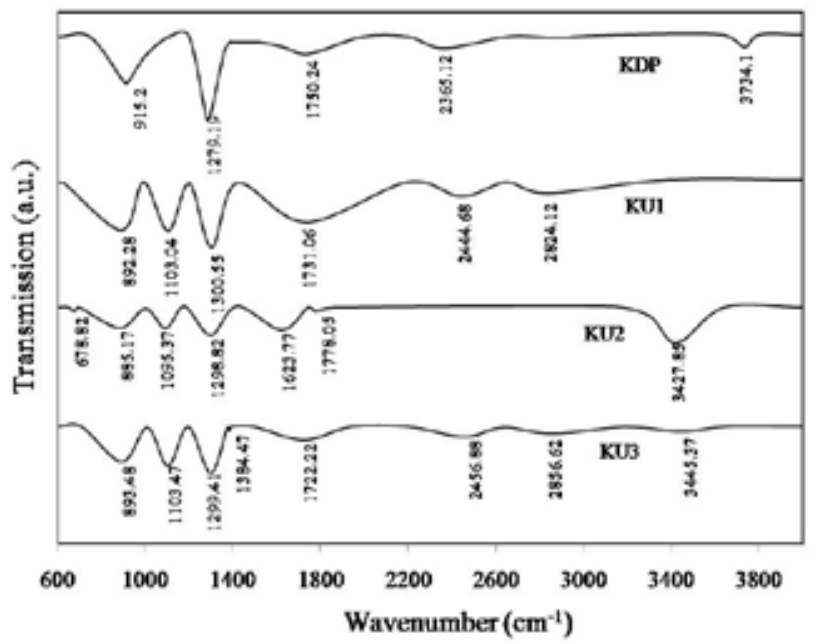

Figure 4. FT-IR spectra of pure and doped KDP crystals

\subsection{UV-Visible-NIR Spectroscopy}

The transmission study on the grown crystal is important. The crystal should be transparent to the fundamental and second harmonic wavelength in order to have use in Second Harmonic Generation (SHG) applications. The transmission of the crystals over wavelength range $190 \mathrm{~nm}-1100 \mathrm{~nm}$ was measured on UV-1700 Shimadzu Spectrophotometer. 3mm thick crystal wafers were cut, polished and used for the transmission study. The increase in the transparency of the doped crystals has been observed. The increase in the doping level improves the fine cutoff at lower wavelength side but no change in the cutoff wavelength has been observed. UV-Vis-NIR spectrums of pure and doped KDP crystals are shown in Figure 5.

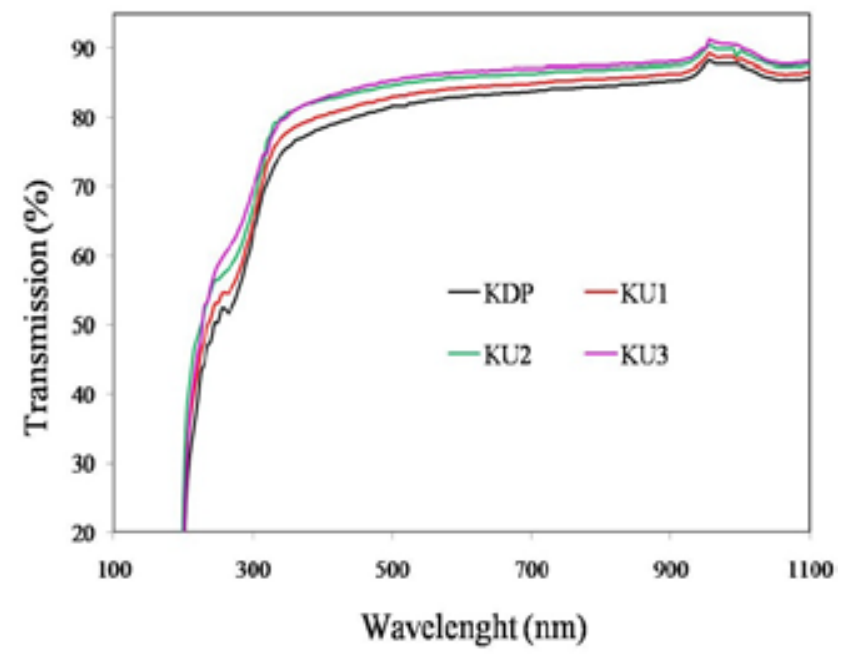

Figure 5. UV-VIS-NIR spectra of pure and doped KDP crystals

\subsection{XRD Study}

The powder XRD patterns (Figure 6) of the pure, 6mol\% UPP doped KDP crystals were recorded using X-ray Diffractometer XPERT-PRO with $\mathrm{Cu} \mathrm{K \alpha}$ radiations (1.54060今́, $40 \mathrm{~mA}, 45 \mathrm{kV}$ ). The powder samples were scanned in steps of $0.0170^{\circ}$ for a time interval of $10.3359 \mathrm{sec}$ over a $2 \theta$ range of $10.0144-119.9874^{\circ}$. The unit cell parameters have been calculated (Table 1) using software Powder X.

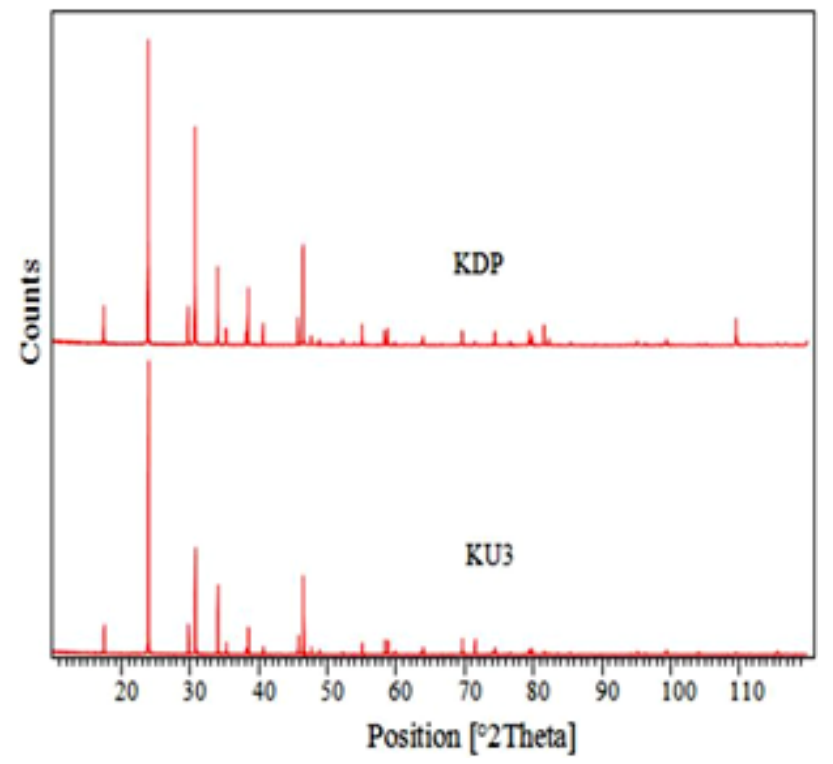

Figure 6. Powder XRD Pattem of KDP and KU3 crystals

Table 1. Unit Cell details

\begin{tabular}{|c|c|c|c|}
\hline & Crystal System & Space Group & Unit Cell Parameters \\
\hline KDP & Tetragonal & I-42d & $\begin{array}{c}\mathrm{a}=\mathrm{b}=7.455 \AA, \mathrm{c}=6.975 \AA . \\
\alpha=\beta=\gamma=90^{\circ}\end{array}$ \\
\hline KU3 & Tetragonal & I-42d & $\begin{array}{c}\mathrm{a}=\mathrm{b}=7.454 \AA, \mathrm{A}=6.976 \AA . \\
\alpha=\beta=\gamma=90^{\circ}\end{array}$ \\
\hline
\end{tabular}

\subsection{SHG Efficiency Measurement}

Kurtz and Perry method[32] was employed to measure the SHG efficiency of the grown crystals in reference with the pure KDP. In the measurement, Q-switched, mode locked Nd:YAG laser of wavelength $1064 \mathrm{~nm}$ of peak power $2.35 \mathrm{~mJ}$, pulse duration $8 \mathrm{~ns}$ and repetition rate $10 \mathrm{~Hz}$ was used. The output was measured at wavelength $532 \mathrm{~nm}$. The SHG efficiencies of the doped crystals found to be more with reference to pure KDP (Table 2).

Table 2. SHG efficiencies of pure and doped KDP crystals

\begin{tabular}{|c|c|}
\hline Crystal & SHG efficiency \\
\hline KDP & 1 \\
\hline KU1 & 1.32 \\
\hline KU2 & 1.37 \\
\hline KU3 & 1.45 \\
\hline
\end{tabular}

\subsection{Ther mal Study}

The Thermo Gravimetric Analysis (TGA) of KU3 was carried out by recording TGA curve on Perkin Elmer TGDTA at a heating rate of $15^{\circ} \mathrm{C} / \mathrm{min}$ under Argon atmosphere. Pure KDP crystal is found to be stable up to temperature $235^{\circ} \mathrm{C}[13]$. The TGA curve of KU3 (Figure 7) re- 
veals the maximum weight loss is in the temperature range $195-355^{\circ} \mathrm{C}$. Prolonged heating does not produce any loss. The weight loss starts earlier and ends latter as compared to the KDP. This weight loss corresponds to the decomposition of the KDP and Urea Phosphate.

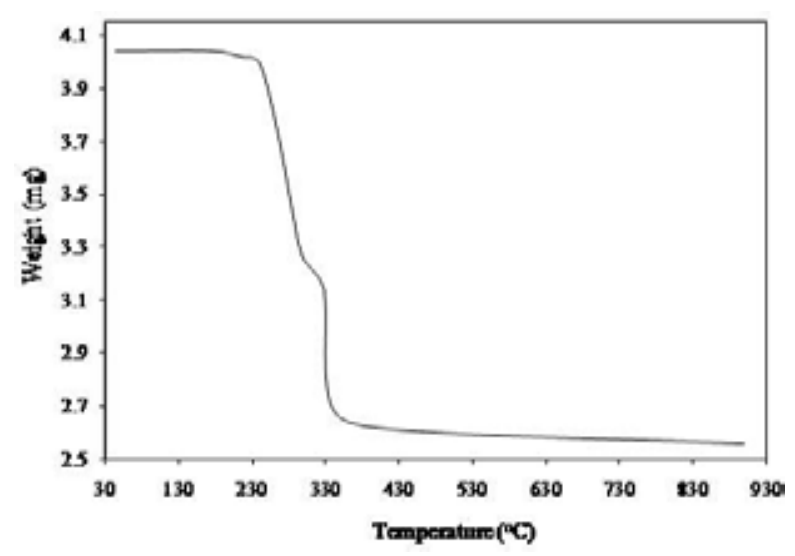

Figure 7. TGA curve of KU3

\section{Conclusions}

The pure and doped KDP crystals with different concentration of Urea Phosphate of best quality were grown from solution within 3-4 weeks.

The doping in the crystals was qualitatively confirmed by the FTIR spectroscopy.

The SHG efficiency study shows the improvement in the efficiency of the doped crystals.

Increasing transparency with concentration of dopant in the host crystal has been confirmed by the UV-Vis-NIR Spectroscopy.

The lattice parameters have been determined from powder XRD study. Doping in the KDP crystals leads to negligible change in the lattice parameters, and the crystal system and space group of the KDP retained.

External morphology of the crystal reveals the decrease in the growth rate along[001] direction in all doped KDP crystals. The growth rate decreases with increasing doping level. This decrease in the growth rate is attributed to the attachment of the bigger size anions on pyramidal faces.

The KU3 crystal is thermally stable up to $195^{\circ} \mathrm{C}$.

\section{ACKNOWLEDGEMENTS}

Author acknowledges the support of Prof. P. K. Das, IPC, IISc Bangaluru for extending the SHG efficiency measurement facility.

\section{REFERENCES}

[1] O. Shimomura, Fast growth and evaluation of potassium dihydrogen phosphate single crystals by electrodialysis with
pH control, J. of Cry. Growth 144 (1994) 253-257.

[2] Atsushi Yokotani, Hiroshi Koide, Takatomo Sasaki, Tatsuhiko Yamanaka and Chiyoe Yamanaka, Fast growth of KDP single crystals by electrodialysis method, J. of Cry. Growth 67 (1984) 627-632.

[3] Guohui Li, Genbo Su, Xinxin Zhuang, Zhengdong Li, Youping He, Rapid growth of KDP cry stal with new additive, J. of Cry. Growth 269 (2004) 443-447.

[4] Masahiro Nakatsuka, Kana Fujioka, Tadashi Kanabe, Hisanori Fujita, Rapid growth over $50 \mathrm{~mm} /$ day of water-soluble KDP crystal, J. of Cry. Growth 171 (1997) 531-537.

[5] N. Zaitseva, L. Carman, I. Smolsky, R. Torres, M. Yan, The effect of impurities and supersaturation on the rapid growth of KDP crystals, J. of Cry. Growth 204 (1999) 512-524.

[6] J. Podder, The study of impurities effect on the growth and nucleation kinetics of potassium dihydrogen phosphate, J. of Cry. Growth 237-239 (2002) 70-75.

[7] Xun Sun, Xinguang Xu, Zhangshou Gao, Youjun Fu, Shenglai Wang, Hong Zeng, Yiping Li, Effect of EDTA on the light scatter in KDP crystal, J. of Cry. Growth 217 (2000) 404-409.

[8] K. Srinivasan, K. Meera, P. Ramasamy, “A contemporary method to enhance the metastable zone width for crystal growth from solution”, Mat. Sc. and Engg. B84 (2001) 233-236

[9] Shouji Hirota, Hideo Miki, Keisuke Fukui, Kouji Maeda, Coloring and habit modification of dyed KDP crystals as functions of supersaturation and dye concentration, J. of Cry. Growth 235 (2002) 541-546.

[10] Selemani Seif, Kamala Bhat, Ashok K. Batra, Mohan D. Aggarwal, Ravindra B. Lal, Effect of Cr(III) impurity on the growth kinetics of potassium dihydrogen phosphate and trigly cine sulfate crystals grown from aqueous solutions, Mat. Letters 58 (2004) 991-994.

[11] I. Owczarek, K. Sangwal, Effect of impurities on the growth of KDP crystals: Mechanism of adsorption on (101) faces, J. of Cry. Growth 102 (1990) 574.

[12] P. Kumaresan, S. Moorthy Babu, P.M. Anbarasan, “Thermal, dielectric studies on pure and amino acid (L-glutamic acid, L-histidine, L-valine) doped KDP single crystals”, Opt. Mat. 30 (2008) 1361-1368

[13] G.G.Muley, M.N. Rode and B.H. Pawar, FT-IR, Thermal and NLO Studies on Amino Acid (L-Arginine and L-Alanine) Doped KDP Crystals, Acta Physica Polonica A, No. 6, 116 (2009).

[14] Guohui Li, Genbo Su, Xinxin Zhuang, Zhengdong Li, Youping He, Rapid growth of KDP cry stal with new additive, J. of Cry. Growth 269 (2004) 443-447.

[15] Ardoino M., Zeng L., Razzetti C., Zha M., Zanotti L., Curti M., Growth and evaluation of some urea derivative crystals, Mat. Chem. and Phy. 66 (2000) 299-302.

[16] V.K. Dixit, S. Vanishri, H.L. Bhat, E. de Matos Gomes, M. Belsley, C. Santinha, G. Arunmozhi, V. Venkataramanan, F. Proena, A. Criado, Crystal growth and characterization of a new nonlinear optical material: Urea l-Malic Acid, J. of Cry. Growth 253 (2003) 460-466. 
[17] Deepthy A., Vanishri S., Ambika D., George Sajan D., Nampoori V.P.N., Bhat H.L.; de Matos Gomes E., Belsley Photoacoustic investigations on thermal anisotropy in urea l-malic acid single crystals, Mat. Res. Bull. 43 (2008)1641-1648.

[18] Li Zhu, Jiayu Zhang, Dingan Chen, Xueyuan Feng, Yonghong $\mathrm{Hu}$, Lingling Xu, Wen Wang, Yiping Cui, Characteristics of an organic nonlinear optical material urea l-malic acid film, Mat. Letters 60 (2006) 1740-1743.

[19] I. Pritula, A. Kosinova, M. Kolybayeva, V. Puzikov, S. Bondarenko, V. Tkachenko, V. Tsurikov, O. Fesenko, Optical, structural and microhardness properties of KDP crystals grown from urea-doped solutions, Mat. Res. Bull. 43 (2008) 2778-2789.

[20] S. Goma, C.M. Padma, C.K. Mahadevan, Dielectric parameters of KDP single crystals added with urea, Mat. Lett. 60 (2006) 3701-3705.

[21] N.P. Rajesh, V. Kannan, P. Santhana Raghavan, P. Ramasamy, C.W. Lan, Optical and microhardness studies of KDP cry stals grown from aqu eous solutions with organic additives, Mat. Lett. 52 (2002) 326-328.

[22] G. G. Muley, M. N. Rode, B. H. Pawar, On the Growth Habit of NLO N, N’ Dimethyl Urea Doped KDP Crystals, Optoele. And Adv. Mat. - Rap. Comm. 3 (2009) 704-706.

[23] K. D. Parikh, D. J. Dave, B. B. Parekh, M. J. Joshi, Growth and characterization of L-alanine doped KDP crystals, Cry. Research and Technology 45 (2010) 603-610.

[24] N. Kanagathara, G. Anbalagan, Growth, Optical and Dielectric Studies on Pure and L-Lysine Doped KDP Crystals, International Journal of Optics 2012 (2012) 1-6.
[25] P. Jagdish, N. P. Rajesh, Effect of L-proline on the growth and NLO properties of KDP crystal, J. of Optoele. And Adv. Mat. 13 (2011) 962 - 966.

[26] You-Jun Fu, Zhang-Shou Gao, Xun Sun, Shen-Lai Wang, Yi-ping Li, Hong Zeng, Jian-Ping Luo, Ai-Dong Duan, Ji-Yang Wang, Effects of Anions on Rapid Growth and Growth Habit of KDP Crystals, Prog. Cry. Gr. and Char. 40 (2000) 211-220.

[27] Y. Wentao, L. Megkai, M. Fanqing, Crystal and molecular structure of urea-(+) tartaric acid, Mat. Res. Bull. 31 (9) (1996) 1127.

[28] J.M.Halbout, A. Sarhangi and C.L.Tang, Nonlinear optical properties of N, N' dimethylurea, Appl. Phys. Lett. 37(1980).

[29] Shouji Hirota, Hideo Miki, Keisuke Fukui, Kouji Maeda, Coloring and habit modification of dyed KDP crystals as functions of supersaturation and dye concentration, J. of Cry. Growth 235 (2002) 541-546.

[30] Selemani Seif, Kamala Bhat, Ashok K. Batra, Mohan D. Aggarwal, Ravindra B. Lal, Effect of Cr(III) impurity on the growth kinetics of potassium dihydrogen phosphate and trigly cine sulfate crystals grown from aqueous solutions, Mat. Lett. 58 (2004) 991- 994.

[31] Tiffany N. Thomas, Terry A. Land, Michael Johnson, William H. Casey, Molecular properties of adsorbates that affect the growth kinetics of archerite (KDP), J. of Coll. and Interface Sc. 280 (2004) 18-26.

[32] S.K. Kurtz, T.T. Perry, A Powder Technique for the Evaluation of Nonlinear Optical Materials, J. Appl. Phys. 39 (1968) 3798. 